\title{
Three-dimensional bulk metamaterials operating in the terahertz range
}

\author{
Fumiaki Miyamaru, ${ }^{1,2, a)}$ Shiro Kuboda, ${ }^{1}$ Kazuo Taima, ${ }^{3}$ Keisuke Takano, ${ }^{4}$ \\ Masanori Hangyo, ${ }^{4}$ and Mitsuo Wada Takeda ${ }^{1}$ \\ ${ }^{1}$ Department of Physics, Faculty of Science, Shinshu University, Matsumoto, Nagano 390-8621, Japan \\ ${ }_{3}^{2}$ PRESTO, Japan Science and Technology Agency, Aoba, Sendai 980-8577, Japan \\ ${ }^{3}$ Fujimori Kogyo Co., Ltd., Chuo, Tokyo 103-0002, Japan \\ ${ }^{4}$ Institute of Laser Engineering, Osaka University, 2-6 Yamadaoka, Suita, Osaka 565-0871, Japan
}

(Received 20 November 2009; accepted 29 January 2010; published online 22 February 2010)

\begin{abstract}
Three-dimensional bulk metamaterials that operate in the terahertz $(\mathrm{THz})$ frequency range were fabricated by stacking 100 two-dimensional sheets containing metallic split-ring resonators (SRR) on thin polyethylene terephthalate film substrates. The THz magnetic resonance for the incident magnetic field perpendicular to the plane of the SRR structure was measured. We also investigated the dependence of the magnetic resonant strength on the metal thickness. (C) 2010 American Institute of Physics. [doi:10.1063/1.3327830]
\end{abstract}

Metamaterials have a great potential for making electromagnetic device components that cannot be obtained using conventional materials. By controlling their electrical permittivity and magnetic permeability, ${ }^{1}$ fascinating electromagnetic properties can be achieved, e.g., a perfect lens with a negative refractive index ${ }^{2}$ and transformation optics. ${ }^{3}$

Metamaterials $^{4-6}$ and their applications ${ }^{7,8}$ have been widely studied in the terahertz $(\mathrm{THz})$ frequency range. Most of those studies were limited to two-dimensional (2D) sheets, whereas three-dimensional (3D) bulk metamaterials whose size is much larger than the wavelength of the operating electromagnetic waves are needed for practical applications. However, the fabrication of such 3D bulk metamaterials is still a considerable challenge and only a few methods have been reported for the fabrication of $3 \mathrm{D} \mathrm{THz}$ metamaterials. ${ }^{9,10}$ A significant hurdle for the fabrication of 3D metamaterials is the appropriate use of lithography, i.e., electron-beam lithography or photolithography, on a substrate made of thick glass or semiconductor wafer. 3D metamaterials can be fabricated by stacking 2D sheets but using a thick glass or semiconductor substrate makes the optical path length in the substrate greater than the desired interlayer distance. Consequently, the effective permittivity and permeability are poorly defined. A true 3D metamaterial requires that the dimensions of the unit cell be smaller than the operating wavelength $\lambda_{\mathrm{op}}$ in each of the three dimensions. The thickness of each layer in a multilayer stacked system must therefore be less than $\lambda_{\text {op }}$.

In this paper, we report the fabrication of 3D bulk metamaterials operating in the $\mathrm{THz}$ region. Our 3D metamaterials consist of 100 layers of 2D sheets of metamaterial patterned with a metallic split-ring resonator (SRR) array on $100-\mu \mathrm{m}$-thick polyethylene terephthalate (PET) film substrates. We investigate the magnetic resonant characteristics of the 3D metamaterials for an incident magnetic field $(\mathbf{H})$ perpendicular to the plane of the SRR structure. We also investigate the dependence of the magnetic resonant strength of the metamaterials on the metal thickness.

The pattern of the SRR array, fabricated by a laser processing on the glass substrate, is transferred to a 70-nm-thick

${ }^{a)}$ Electronic mail: miyamaru@ @hinshu-u.ac.jp. silver layer on the commercially available $100-\mu$ m-thick PET films by chemical reduction. The refractive index of the PET film is measured to be $n_{\mathrm{PET}}=1.56$ at $0.5 \mathrm{THz}$. After the reductive deposition of the silver layer, heat treatment increases its conductivity. Non-electrolytic plating with copper can increase the conductivity even further. After this process, the total metal thickness is $0.7-15 \mu \mathrm{m}$. A microscope image of one of our sample is shown in Figs. 1(a) and 1(b). The spatial resolution of our fabrication process is about $10 \mu \mathrm{m}$. The SRRs, of length $L=100 \mu \mathrm{m}$, are periodically arranged in a square lattice structure with periodicity $p=170 \mu \mathrm{m}$. The gap in the SRRs and the line width are about 30 and $15 \mu \mathrm{m}$,

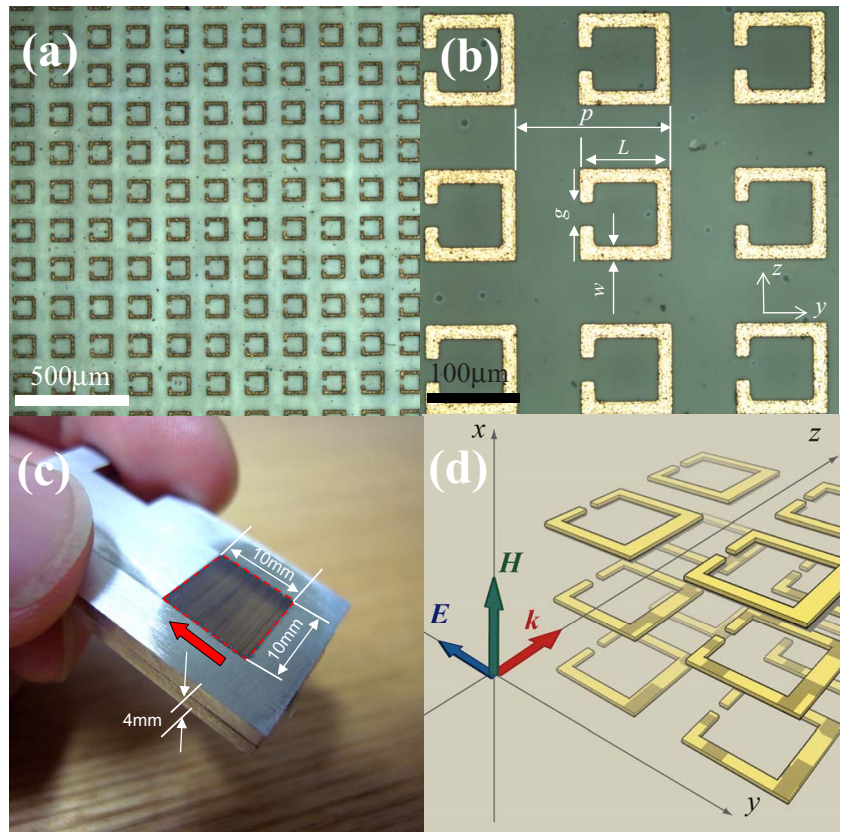

FIG. 1. (Color online) (a) Microscope image of SRR structures fabricated on the PET film. (b) Enlarged image of the SRR structures. The electromagnetic characteristics are determined by geometrical parameters: in-plane unit cell size $p$, SSR size $L$, gap width $g$, and line width $w$. (c) A photograph of a $3 \mathrm{D}$ bulk metamaterial. A red arrow shows the stacked direction of $2 \mathrm{D}$ metamaterial sheets. This metamaterial contains about 180000 unit cells. (d) A schematic configuration of the incident electromagnetic wave and the SRR arrays for inducing the magnetic resonance with an incident magnetic field $\mathbf{H}$. 


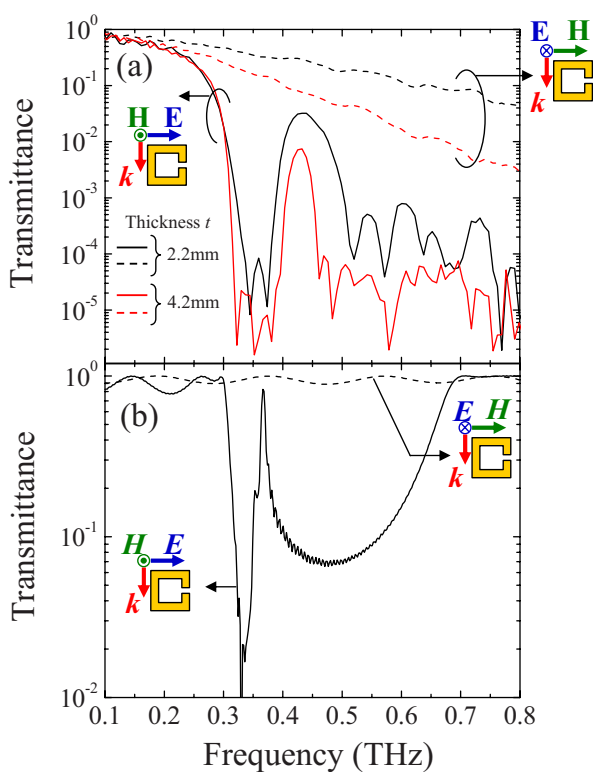

FIG. 2. (Color online) (a) Measured transmission spectra for the 3D metamaterials of thickness $t=2.2 \mathrm{~mm}$ (black lines) and $t=4.4 \mathrm{~mm}$ [gray (red) lines] in the propagation direction. Solid and dashed lines are for the incident field $\mathbf{H}$ perpendicular and parallel to the SRR plane, respectively. (b) Simulated transmission spectra obtained by a FDTD method. Solid and dashed lines are for the same optical configuration as those in (a).

respectively. The 3D metamaterial is made by stacking 100 layers of such 2D lattices. Figure 1(c) shows one of our 3D bulk metamaterials with size $10 \times 10 \times 4 \mathrm{~mm}^{3}$ and involving about 180000 unit cells. In the 3D metamaterial, the layers are held together mechanically with an aluminum frame. Since the individual SRRs show a bianisotropic response to an incident electric field $\mathbf{E}$ perpendicular to the gap, ${ }^{11,12}$ all the SRRs in all the layers must be aligned in the same direction. By making the incident field $\mathbf{E}$ parallel to the gap, as shown in Fig. 1(d), we observe only the response to the incident $\mathbf{H}$ field perpendicular to the SRR plane. In a previous study, ${ }^{13}$ we found that there is no significant contribution from the interlayer interaction to the transmission characteristics. This allows us to stack 2D metamaterial sheets without the need for a precise lateral alignment between layers. To observe the electromagnetic properties of the metamaterials, we used a $\mathrm{THz}$ time-domain spectroscopic system, which allows us to obtain the waveform of a $\mathrm{THz}$ wave directly in the time domain. By means of Fourier transformations, we can calculate the transmission and phase spectra from the time-domain waveforms with and without samples.

We first investigate magnetic resonance in the 3D metamaterial. In our previous study, ${ }^{13}$ we measured the transmission spectra of 2D metamaterial sheets consisting of SRR arrays, and observed the magnetic resonance at oblique incidence. For the 3D metamaterials, we can directly measure the magnetic response of the SRR array. Figure 2(a) shows the measured transmission spectrum (solid black line) of the 3D metamaterial with a thickness of $t=2.2 \mathrm{~mm}$ in the propagation direction. The optical configuration is shown on the left hand side of Fig. 2(a), where the incident $\mathbf{H}$ is directed perpendicular to the SRR plane. The incident $\mathbf{E}$ is directed parallel to the gap in order to reduce the bianisotropic effect due to the SRR structure. ${ }^{11,12}$ A resonant transmission dip is observed clearly around $0.35 \mathrm{THz}$. For comparison, the transmission spectrum for an incident $\mathbf{H}$ parallel to the SRR plane is also plotted in Fig. 2(a) (dashed black line). In this configuration, no resonant dip is observed. These results indicate that our sample works as a 3D metamaterial with magnetic resonance in the $\mathrm{THz}$ frequency range. We perform a computational simulation of the transmission response with a finite difference time domain (FDTD) method for comparison with our experimental observations. Figure 2(b) shows the calculated transmission spectra for the SRR array with the same geometrical parameters. The resonant transmission dip is clearly observed around $0.34 \mathrm{THz}$, in good agreement with the experiment (solid line). The broader dip, centered around $0.47 \mathrm{THz}$, results from the halfwavelength electric resonance of one side of the SRR. ${ }^{12}$ The intensity of the magnetic resonant dip for the 3D metamaterial with $t=2.2 \mathrm{~mm}$ seems to be beyond the dynamic range of our spectroscopic system. Such a saturation of the transmission dip intensity makes it difficult to estimate the effective permeability of the 3D metamaterial. This can be confirmed by measuring the transmission spectrum for the 3D metamaterial with $t=4.4 \mathrm{~mm}$, as shown in Fig. 2(a). The intensity of magnetic resonant dip for the incident $\mathbf{H}$ perpendicular to the SRR plane (solid red line) is almost the same as that of the 3D metamaterial with $t=2.2 \mathrm{~mm}$, while the overall transmittance for the incident $\mathbf{H}$ parallel to the SRR plane (dashed red line) decreases with thickness $t$. These results indicate that the intensity of the magnetic resonant dip is saturated for $t=2.2 \mathrm{~mm}$ as a result of the limited dynamic range of our spectroscopic system. In Fig. 2(a), overall transmittance decreases with increasing the frequency for all spectra. Since the absorption coefficient of PET increases with frequency, the decrease of the transmittance with the frequency is attributed to the absorption of the PET film substrate. In our simulation, we did not involve the absorption of PET for simplicity. In that case, the transmittance does not decrease with frequency, which is different from the experimental transmittance. These results indicate that the gradual decrease in the transmittance with frequency is due to the absorption of the PET substrate.

Next, we investigate the incident-angle dependence of the 3D metamaterial. For negative-index 3D metamaterials, the angular dispersion is an important characteristic. ${ }^{14}$ Figures 3(a)-3(d) show the measured transmission spectra of the $3 \mathrm{D}$ metamaterial at various incident angles ranging from $0^{\circ}$ to $45^{\circ}$. The magnetic resonant frequency shows only slight change as the incident angle increases. This very low angular dispersion demonstrates that the observed resonant dip results from the resonance in each unit cell. In other words, there is little contribution from multiple interferences in the periodic structure.

The magnetic resonant strength is a crucial characteristic for achieving a SRR with a negative effective permeability and a negative refractive index. If the silver layer is thin compared to the skin depth of electromagnetic waves (i.e., a few hundred nanometers) at this frequency, the resonant strength is then too weak and the effective permeability does not become negative. We investigate the dependence of the magnetic resonant strength on the metal thickness. Since the intensity of the magnetic resonant dip is saturated for 3D metamaterials, as observed in Fig. 2(a), in this experiment we measured the transmission spectra of one layer of the 2D metamaterial sheet at normal incidence. The relation between the electric field and the SRR is shown in the inset of Fig. 4(a). In this configuration, we observe the resonant dip at- 


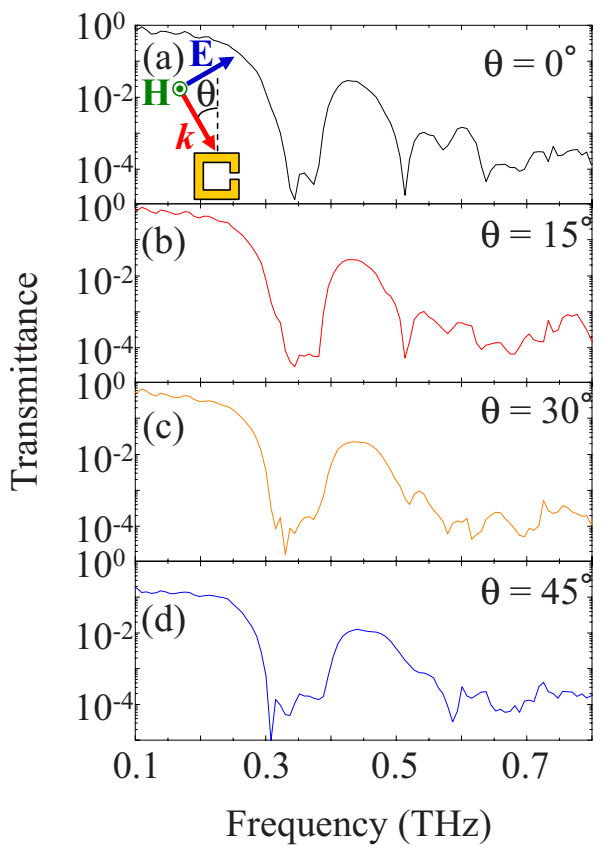

FIG. 3. (Color online) Measured transmission spectra of 3D metamaterials at incident angles $\theta$ of (a) $0^{\circ}$, (b) $15^{\circ}$, (c) $30^{\circ}$, and (d) $45^{\circ}$ for the incident $\mathbf{H}$ perpendicular to the SRR plane. The relation between the incident $\mathrm{THz}$ wave and the SRR is shown in the inset of (a).

tributed to the bianisotropic effect ${ }^{12}$ at nearly the same frequency as that of the magnetic resonance observed in $3 \mathrm{D}$ metamaterials [Fig. 2(a)]. Figures 4(a)-4(d) show the measured transmission spectra of the 2D metamaterial sheets with different metal thicknesses $h(h=0.07,0.7,5$, and
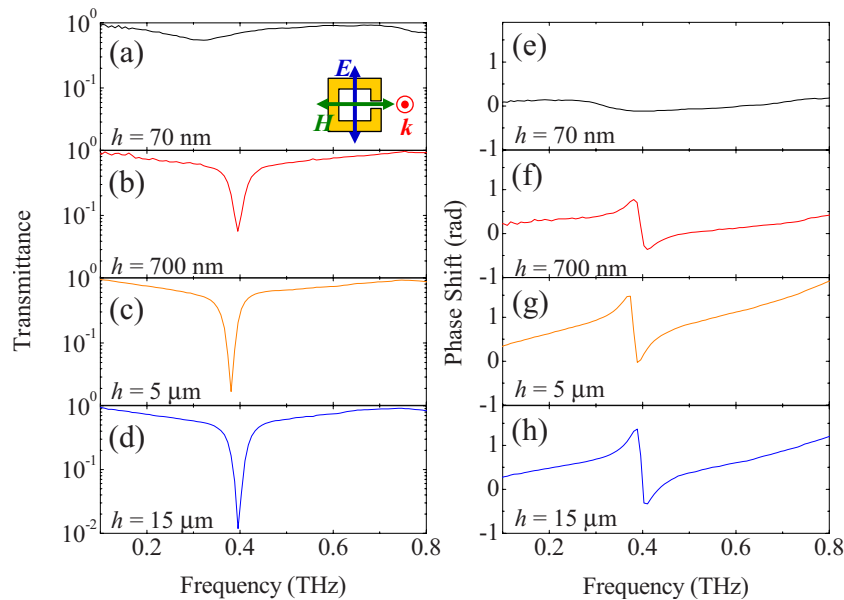

FIG. 4. (Color online) Measured [(a)-(d)] transmission and [(e)-(h)] phase shift spectra of one layer of $2 \mathrm{D}$ metamaterial sheets at normal incidence for different metal thicknesses $h$ [(a) and (e) $h=0.07 \mu \mathrm{m}$, (b) and (f) $h$ $=0.7 \mu \mathrm{m},(\mathrm{c})$ and (g) $h=5 \mu \mathrm{m}$, (d) and (h) $h=15 \mu \mathrm{m}]$.
$15 \mu \mathrm{m})$. Figures 4(e)-4(h) show the corresponding measured phase-shift spectra. For $h=0.07 \mu \mathrm{m}$ in Fig. 4(a), the weak resonant dip is observed around $0.36 \mathrm{THz}$. The corresponding phase modulation around the resonant frequency is also weak, as shown in Fig. 4(e). The strength of the magnetic resonance increases with the metal thickness, as shown in Figs. 4(b)-4(d), and tends to saturate around $h=5 \mu \mathrm{m}$ where the metal thickness is thick compared to the skin depth. These results agree well with the qualitative ones given in Ref. 15. In the phase-shift spectra, the phase modulation around the resonant frequency becomes stronger with increasing metal thickness. Such a strong phase modulation indicates the possibility to achieve a negative effective permeability around the resonant frequency.

In conclusion, we fabricated 3D bulk metamaterials operating in the $\mathrm{THz}$ region by stacking 100 sheets of $2 \mathrm{D}$ metamaterial, each containing a lattice of metallic SRR structures. We measured the transmission spectrum of the 3D metamaterials and confirmed a magnetic resonance, attributed to the resonance localized in each unit cells that occurs for an incident $\mathbf{H}$ perpendicular to the SRR plane. These are promising results for making $3 \mathrm{D}$ bulk materials with a negative refractive index.

This work was supported partially by the Ministry of Education, Science, Sports and Culture, Grant-in-Aid for Scientific Research (A) No. 20244047, and for Young Scientists (B) No. 21760036.

${ }^{1}$ J. B. Pendry, A. J. Holden, D. J. Robbins, and W. J. Stewart, IEEE Trans. Microwave Theory Tech. 47, 2075 (1999).

${ }^{2}$ J. B. Pendry, Phys. Rev. Lett. 85, 3966 (2000).

${ }^{3}$ J. B. Pendry, D. Schurig, and D. R. Smith, Science 312, 1780 (2006).

${ }^{4}$ T. Yen, W. Padilla, N. Fang, D. Vier, D. Smith, J. B. Pendry, D. N. Basov, and X. Zhangm, Science 303, 1494 (2004).

${ }^{5}$ S. Linden, C. Enkrich, M. Wegener, J. Zhou, and T. Koschny, Science 306, 1351 (2004).

${ }^{6}$ F. Miyamaru, Y. Saito, M. W. Takeda, B. Hou, L. Liu, W. Wen, and P. Sheng, Phys. Rev. B 77, 045124 (2008).

${ }^{7}$ H.-T. Chen, W. J. Padilla, J. M. O. Zide, A. C. Gossard, A. J. Taylor, and R. D. Averitt, Nature (London) 444, 597 (2006).

${ }^{8}$ J. O'Hara, R. Singh, I. Brener, E. Smirnova, and J. Han, Opt. Express 16, 1786 (2008).

${ }^{9}$ B. Casse, H. Moser, J. Lee, M. Bahou, and S. Inglis, Appl. Phys. Lett. 90, 254106 (2007).

${ }^{10}$ O. Paul, C. Imhof, B. Reinhard, R. Zengerle, and R. Beigang, Opt. Express 16, 6736 (2008).

${ }^{11}$ R. Marqués, F. Medina, and R. Rafii-El-Idrissi, Phys. Rev. B 65, 144440 (2002).

${ }^{12}$ W. J. Padilla, A. J. Taylor, and R. D. Averitt, Phys. Rev. Lett. 96, 107401 (2006).

${ }^{13}$ F. Miyamaru, M. W. Takeda, and K. Taima, Appl. Phys. Express 2, 042001 (2009).

${ }^{14}$ R. A. Shelby, D. R. Smith, and S. Schultz, Science 292, 77 (2001).

${ }^{15}$ R. Singh, E. Smirnova, A. J. Taylor, J. F. O'Hara, and W. Zhang, Opt. Express 16, 6537 (2008). 